\title{
COVERING CERTAIN MONOLITHIC GROUPS WITH PROPER SUBGROUPS
}

\author{
MARTINO GARONZI
}

\begin{abstract}
Given a finite non-cyclic group $G$, call $\sigma(G)$ the least number of proper subgroups of $G$ needed to cover $G$. In this paper we give lower and upper bounds for $\sigma(G)$ for $G$ a group with a unique minimal normal subgroup $N$ isomorphic to $A_{n}^{m}$ where $n \geq 5$ and $G / N$ is cyclic. We also show that $\sigma\left(A_{5} \imath C_{2}\right)=57$.
\end{abstract}

\section{INTRODUCTION}

Given a finite non-cyclic group $G$, call $\sigma(G)$ the least number of proper subgroups of $G$ needed to cover $G$ set-theoretically. This notion has been introduced the first time by Cohn in 1994 in [5]. We usually call "cover" of $G$ a family of proper subgroups of $G$ which covers $G$, and "minimal cover" of $G$ a cover of $G$ consisting of exactly $\sigma(G)$ elements. If $G$ is cyclic then $\sigma(G)$ is not well defined because no proper subgroup contains any generator of $G$; in this case we define $\sigma(G)=\infty$, with the convention that $n<\infty$ for every integer $n$. In [15] Tomkinson showed that if $G$ is a finite solvable group then $\sigma(G)=q+1$, where $q$ is the least order of a chief factor of $G$ with more than one complement. The behavior of the function $\sigma$ has been intensively studied for the almost simple groups. The alternating and symmetric groups have been considered by Maróti in [12. In [2] Britnell, Evseev, Guralnick, Holmes and Maróti studied the linear groups $G L(n, q)$, $P G L(n, q), S L(n, q), P S L(n, q)$. In [10] Lucido studied the Suzuki groups. In 9] Lucchini and Maróti found an asymptotic formula for the function which assigns to the positive integer $x$ the number of positive integers $n$ at most $x$ with the property that $\sigma(S)=n$ for some non-abelian simple group $S$.

If $N$ is a normal subgroup of a finite group $G$ then $\sigma(G) \leq \sigma(G / N)$, since every cover of $G / N$ can be lifted to a cover of $G$. We say that $G$ is " $\sigma$-primitive" if $\sigma(G)<\sigma(G / N)$ for every non-trivial normal subgroup $N$ of $G$. Since every finite group has a $\sigma$-primitive epimorphic image with the same $\sigma$, the structure of the $\sigma$-primitive groups is of big interest. It was studied by Lucchini and Detomi in 8 . They proved for instance that every $\sigma$-primitive group is a subdirect product of monolithic groups (i.e. groups with only one minimal normal subgroup). This and other partial results lead us to believe that the monolithic groups have a crucial role in this story. In the same paper Lucchini and Detomi conjectured that every non-abelian $\sigma$-primitive group is monolithic. This motivates us in the study of the function $\sigma$ for the monolithic $\sigma$-primitive groups.

Let us consider a monolithic $\sigma$-primitive group $G$. If $\operatorname{soc}(G)$ is abelian then it is easy to prove that $\operatorname{soc}(G)$ is complemented in $G$ and $\sigma(G)=c+1$, where $c$ is the number of complements of $\operatorname{soc}(G)$ in $G$. Let now $n, m$ be positive integers

Date: 27th of August, 2011. 
with $n \geq 5$. Suppose that $\operatorname{soc}(G)=A_{n}^{m}$ and that $G / \operatorname{soc}(G)$ is cyclic. Write $\operatorname{soc}(G)=T_{1} \times \cdots \times T_{m}=T^{m}$, with $T=A_{n}$, and define $X:=N_{G}\left(T_{1}\right) / C_{G}\left(T_{1}\right)$. Then either $X \cong A_{n}$ ("even case") or $X \cong S_{n}$ ("odd case"). In the even case $G \cong A_{n} \succ C_{m}$ (cfr. [1], Definition 1.1.8 and Remark 1.1.40.13). These groups have been studied in [13] obtaining lower and upper bounds for $\sigma(G)$ and its exact value in the case $n \equiv 2 \bmod (4)$.

Consider now the odd case. Let $\gamma \in G$ be such that $\gamma \operatorname{soc}(G)$ generates $G / \operatorname{soc}(G)$, so that $G=\left\langle T^{m}, \gamma\right\rangle$. Since

$$
T^{m}<G \leq \operatorname{Aut}\left(T^{m}\right) \cong T \text { <ym }(m),
$$

every element of $G$ has the form $\left(x_{1}, \ldots, x_{m}\right) \gamma^{k}$ with $x_{1}, \ldots, x_{m} \in T$ and $k$ an integer. Moreover $\gamma$ itself is of the form $\left(y_{1}, \ldots, y_{m}\right) \delta$ with $y_{1}, \ldots, y_{m} \in \operatorname{Aut}\left(A_{n}\right)$, and $\delta \in \operatorname{Sym}(m)$ is an $m$-cycle since $G$ acts transitively on the $m$ factors of $T^{m} \cdot \gamma$ can be chosen in such a way that each $y_{i}$ is either 1 or equal to $\tau:=(12) \in S_{n}-A_{n}$. Since we are in the odd case the number of indices $i \in\{1, \ldots, m\}$ such that $y_{i}=\tau$ is odd. It is easy to show that $\gamma$ is conjugate to $(1, \ldots, 1, \tau) \delta$ in $G$. Therefore we may choose $\gamma$ to be $(1, \ldots, 1, \tau) \delta$ and clearly it is not restrictive to choose $\delta:=(1 \cdots m)$. It turns out that $G$ is the semidirect product

$$
A_{n}^{m} \rtimes\langle\gamma\rangle .
$$

Let us fix some notation. Let $C:=C_{G}\left(T_{1}\right)$. Let $U$ be a maximal subgroup of $G$ supplementing the socle $N$ of $G$. $U$ is called "of product type" if $U=N_{G}(M \times$ $\left.M^{a_{2}} \times \cdots \times M^{a_{m}}\right)$ with $M$ a maximal $N_{U}\left(T_{1}\right)$-invariant subgroup of $T_{1}$ (cfr. [1, Remark 1.1.40.20) and $a_{2}, \ldots, a_{m} \in \operatorname{Aut}\left(A_{n}\right)$. In this case $M=N_{U}\left(T_{1}\right) \cap T_{1}$ and $N_{U}\left(T_{1}\right) C / C$ is a maximal subgroup of $N_{G}\left(T_{1}\right) / C \cong S_{n}$ (cfr. [1], Remark 1.1.40.21) whose intersection with $T_{1} C / C$ is $M C / C \cong M$, so that $M$ is of the form $K \cap A_{n}$ with $K$ maximal in $S_{n}$. $U$ is said to be of "diagonal type" if $U=N_{G}(\Delta)$ where $\Delta=$ $\Delta_{1} \times \cdots \times \Delta_{m / q}$, where $q$ is a prime divisor of $m$ and $\Delta_{i}=\left\{\left(x, x^{\alpha_{i_{1}}}, \ldots, x^{\alpha_{i_{q}}}\right) \mid x \in\right.$ $\left.A_{n}\right\}$, where $\alpha_{i_{k}} \in \operatorname{Aut}\left(A_{n}\right)$ for $k=1, \ldots, q$. In this case we also say that $U$ is of "diagonal type $q$ ". It turns out that every maximal subgroup of $G$ supplementing the socle is either of product type or of diagonal type.

In this paper we establish the following result, generalizing the results in 12 about $\sigma\left(S_{n}\right)$ (which corresponds to the case $m=1$ ). The arguments we use involve the same covers of $S_{n}$ considered in [12, and this is why the results have similar flavour: in particular, we obtain an exact formula for $\sigma(G)$ when $n$ is odd with some exceptions, and an asymptotic formula when $n$ is even.

Theorem 1. Let $m, n$ be positive integers, and let $G:=A_{n} \rtimes C_{2 m}$ as above. Let $\omega(x)$ denote the number of prime factors of the positive integer $x$. The following holds.

(1) Suppose that $n \geq 7$ is odd and $m \neq 1$ if $n=9$. Then

$$
\sigma(G)=\omega(2 m)+\sum_{i=1}^{(n-1) / 2}\left(\begin{array}{l}
n \\
i
\end{array}\right)^{m} .
$$

(2) If $n=5$ then

$$
10^{m} \leq \sigma(G) \leq \omega(2 m)+5^{m}+10^{m} .
$$

If $n=5$ and every prime divisor of $m$ is either 2 or 3 then

$$
\sigma(G)=\omega(2 m)+5^{m}+10^{m} .
$$


(3) Suppose that $n \geq 8$ is even. Then

$$
\begin{aligned}
& \left(\frac{1}{2}\left(\begin{array}{c}
n \\
n / 2
\end{array}\right)\right)^{m} \leq \sigma(G) \leq \omega(2 m)+\left(\frac{1}{2}\left(\begin{array}{c}
n \\
n / 2
\end{array}\right)\right)^{m}+\sum_{i=1}^{[n / 3]}\left(\begin{array}{c}
n \\
i
\end{array}\right)^{m} . \\
& \text { In particular } \sigma(G) \sim\left(\frac{1}{2}\left(\begin{array}{c}
n \\
n / 2
\end{array}\right)\right)^{m} \text { as } n \rightarrow \infty . \\
& \text { (4) If } n=6 \text { then } \sigma(G)=\omega(2 m)+2 \cdot 6^{m} .
\end{aligned}
$$

Here the upper bound for $\sigma(G)$ is always given by the cardinality of a cover consisting of the $\omega(2 m)$ maximal subgroups of $G$ containing its socle and suitable maximal subgroups of product type, $N_{G}\left(M \times M^{a_{2}} \times \cdots \times M^{a_{m}}\right)$, where the $N_{S_{n}}(M)$ 's cover $S_{n}-A_{n}$.

We also compute $\sigma\left(A_{5} C_{2}\right)$ (corresponding to the even case when $(n, m)=(5,2)$ ), which is not computed in 13. Similarly as above and as in the results in 13, a minimal cover of $A_{5} C_{2}$ consists of the maximal subgroups containing the socle and a family of subgroups of product type corresponding to a cover of $A_{5}$ (consisting of the normalizers of the Sylow 5-subgroups and four point stabilizers).

Theorem 2. $\sigma\left(A_{5} 2 C_{2}\right)=1+4 \cdot 5+6 \cdot 6=57$.

Compare this result with the corresponding odd case: $\sigma\left(A_{5}^{2} \rtimes C_{4}\right)=1+5 \cdot 5+$ $10 \cdot 10=126$. Note that $A_{5}<C_{2}$ is the easiest example of a non-almost-simple monolithic group with non-abelian socle.

\section{Preliminary lemmas}

In the present section we collect some technical lemmas which will be useful in the next section.

Let $n$ be a positive integer and let $c_{1}, \ldots, c_{k} \in\{1, \ldots, n\}$ be such that $c_{1}+\ldots+$ $c_{k}=n$. A " $\left(c_{1}, \ldots, c_{k}\right)$-cycle" will be an element of $S_{n}$ which can be written as the product of $k$ pairwise disjoint cycles of length $c_{1}, \ldots, c_{k}$. An "intransitive subgroup of $S_{n}$ (resp. $A_{n}$ ) of type $\left(c_{1}, \ldots, c_{k}\right)$ " will be the biggest subgroup of $S_{n}$ (resp. $A_{n}$ ) acting on $\{1, \ldots, n\}$ with $k$ given orbits of size $c_{1}, \ldots, c_{k}$. It is clearly isomorphic to $S_{c_{1}} \times \cdots \times S_{c_{k}}\left(\operatorname{resp} .\left(S_{c_{1}} \times \cdots \times S_{c_{k}}\right) \cap A_{n}\right)$.

Proposition 1 (Stirling's formula). For all positive integers $n$ we have

$$
\sqrt{2 \pi n}(n / e)^{n} e^{1 /(12 n+1)}<n !<\sqrt{2 \pi n}(n / e)^{n} e^{1 /(12 n)} .
$$

The following lemma is shown in the proof of lemma 2.1 in [11.

Lemma 1. For a positive integer $n$ at least 8 we have

$$
((n / a) !)^{a} a ! \geq((n / b) !)^{b} b !
$$

whenever $a$ and $b$ are divisors of $n$ with $a \leq b$.

Lemma 2. Let $n \neq 9,15$ be an odd positive integer, and let $a \geq 3$ be a proper divisor of $n$. Then

$$
\left(\frac{n-1}{2}\right) !\left(\frac{n-3}{2}\right) ! \geq(n / a) !^{a} \cdot a !
$$


Proof. Proceed by inspection for $21 \leq n \leq 299$, using lemma 1 Assume $n \geq 300$. Let us use Stirling's formula. We are reduced to prove that

$$
\begin{aligned}
\sqrt{\pi(n-1)}((n-1) / 2 e)^{(n-1) / 2} \sqrt{\pi(n-3)}((n-3) / 2 e)^{(n-3) / 2} \geq \\
\geq 2 \sqrt{2 \pi n / a}^{a}(n / a e)^{n} \sqrt{2 \pi a}(a / e)^{a} .
\end{aligned}
$$

Using the inequalities $\pi \geq \sqrt{2 \pi}$ and $n-3 \geq a$ we are reduced to prove that

$$
(n-1)^{1 / 2}(n-1)^{(n-1) / 2}(n-3)^{(n-3) / 2} \geq 2 /(2 e)^{2} \sqrt{2 \pi n / a}^{a}(2 n / a)^{n}(a / e)^{a},
$$

and using $n-1 \geq n-3$ we obtain:

$$
(n-1)^{1 / 2}(n-3)^{n-2} \geq\left(2 /\left(4 e^{2}\right)\right)(2 \pi n / a)^{a / 2}(2 n / a)^{n}(a / e)^{a} .
$$

Using the inequality $3 \leq a \leq \sqrt{n}$ we obtain:

$$
(n-1)^{1 / 2}(n-3)^{n-2} \geq\left(2 / 4 e^{2}\right)(2 \pi n / 3)^{\sqrt{n} / 2}(2 n / 3)^{n}(\sqrt{n} / e)^{\sqrt{n}} .
$$

Take logarithms and divide by $n$, obtaining

$$
\begin{gathered}
(1 / 2 n) \log (n-1)+((n-2) / n) \log (n-3) \geq(1 / n) \log \left(2 / 4 e^{2}\right)+(1 / 2 \sqrt{n}) \log (2 \pi / 3)+ \\
+(1 / 2 \sqrt{n}) \log (n)+\log (2 n / 3)+(1 / \sqrt{n}) \log (\sqrt{n} / e) .
\end{gathered}
$$

Since $\sqrt{n-1} \geq 2 / 4 e^{2}$ and $(1 / 2 \sqrt{n}) \log (2 \pi / 3) \leq 1 / \sqrt{n}$ we are reduced to show that

$$
\log (n-3) \geq(2 / n) \log (n-3)+(1 / \sqrt{n}) \log (n)+\log (2 n / 3) .
$$

Since $n \geq 300$ we have that $(2 / n) \log (n-3)+(1 / \sqrt{n}) \log (n)<0.37$, hence it suffices to show that $\log (n-3) \geq 0.37+\log (2 n / 3)$, i.e. $n-3 \geq(2 / 3) e^{0.37} \cdot n$. This is true since $(2 / 3) e^{0.37}<0.97$.

Corollary 1. Let $n \geq 11$ be an odd integer. Then the order of an intransitive maximal subgroup of $S_{n}$ (resp. $A_{n}$ ) is bigger than the order of any transitive maximal subgroup of $S_{n}$ (resp. $A_{n}$ ) different from $A_{n}$.

Proof. The imprimitive case follows from the lemma noticing that $((n+1) / 2) !((n-$ $1) / 2) ! \geq((n-1) / 2) !((n-3) / 2) !$, and if $n=15$ then $((n+1) / 2) !((n-1) / 2) ! \geq$ $(n / a) !^{a} a$ ! for $a \in\{3,5\}$. By [11] the order of a primitive maximal subgroup of $A_{n}$ or $S_{n}$ is at most $2.6^{n}$ and $((n+1) / 2) !((n-1) / 2) ! \geq 2.6^{n}$.

Lemma 3. Let $n, a, b$ be positive integers, with $a>b$.

(1) Suppose $n$ is odd. Let $K$ be an intransitive maximal subgroup of $A_{n}$. If $\left(n^{2}-1\right)^{a} \geq 4^{a} e^{2(a-b)} n^{2 b}$, then $|K|^{a / b} \geq\left|A_{n}\right|$.

(2) Suppose $n$ is even. Let $K$ be a maximal imprimitive subgroup of $A_{n}$ of the form $\left(S_{n / 2} 2 S_{2}\right) \cap A_{n}$. If $n^{a} \geq 2^{a} e^{a-b} n^{b}$, then $|K|^{a / b} \geq\left|A_{n}\right|$.

Proof. We prove only (1), since the proof of (2) is similar. Suppose $n$ is odd. Since the smallest intransitive maximal subgroups of $A_{n}$ are the ones of type ( $n-$ $1) / 2,(n+1) / 2)$, what we have to prove is the following inequality:

$$
(1 / 2)^{a / b}((n-1) / 2) !^{a / b}((n+1) / 2) !^{a / b} \geq n ! / 2 .
$$

Since $e^{\frac{a / b}{6(n-1)+1}+\frac{a / b}{6(n+1)+1}} \geq e^{1 / 12 n}$ for every positive integer $n$, using Stirling's formula we see that it is sufficient to show that

$$
\begin{gathered}
(1 / 2)^{a / b}((n-1) / 2 e)^{a(n-1) / 2 b} \sqrt{(\pi(n-1))^{a / b}}((n+1) / 2 e)^{a(n+1) / 2 b} \sqrt{(\pi(n+1))^{a / b}} \geq \\
\geq(1 / 2)(n / e)^{n} \sqrt{2 \pi n} .
\end{gathered}
$$


Re-write this as follows:

$$
\begin{gathered}
\left(\left(n^{2}-1\right) / 4 e^{2}\right)^{a(n-1) / 2 b}(\pi / 2)^{a / b}\left(n^{2}-1\right)^{a / 2 b}((n+1) / 2 e)^{a / b} \geq \\
\geq(1 / 2)(n / e)^{n} \sqrt{2 \pi n} .
\end{gathered}
$$

In other words:

$$
\left(\left(n^{2}-1\right) / 4 e^{2}\right)^{a n / 2 b}(\pi(n+1) / 2)^{a / b} \geq(1 / 2) \sqrt{2 \pi n}(n / e)^{n} .
$$

Since $\pi(n+1) / 2 \geq(1 / 2) \sqrt{2 \pi n}$ we are reduced to prove that

$$
\left(\left(n^{2}-1\right) / 4 e^{2}\right)^{a n / 2 b} \geq(n / e)^{n},
$$

i.e.

$$
\left(n^{2}-1\right)^{a} \geq(n / e)^{2 b}\left(4 e^{2}\right)^{a}=4^{a} e^{2(a-b)} n^{2 b} .
$$

Lemma 4. Let $n$ be an odd positive integer at least 5 , let a be a $(2, n-2)$-cycle in $S_{n}$, and let b be a $(n-1)$-cycle in $S_{n}$. No primitive maximal subgroup of $S_{n}$ contains a, no imprimitive maximal subgroup of $S_{n}$ contains $b$, and no intransitive maximal subgroup of $S_{n}$ contains both $a$ and $b$.

Proof. The second and the third statement are clear. If a primitive subgroup of $S_{n}$ contains $a$ then it contains the transposition $a^{n-2}$, thus it contains $A_{n}$ by the Jordan theory (cfr. for example 4, Theorem 6.15 and Exercise 6.6).

In the rest of this section we will use the notations which we fixed in the introduction.

Lemma 5. Let $1 \leq k<2 m$ be an integer coprime to $2 m$. In the following let the subscripts be identified with their reductions modulo $m$, and let $b_{1}:=1, b_{2}, \ldots$, $b_{m} \in S_{n}, x_{1}, \ldots, x_{m} \in A_{n}$. Let $M$ be a subgroup of $A_{n}$. The following holds.

(1) Suppose $k<m$. For $d \in\{1, \ldots, m\}$ define $\tau_{d}$ to be $\tau$ if $d>m-k$, and 1 if $d \leq m-k$. Then the element $\left(x_{1}, \ldots, x_{m}\right) \gamma^{k} \in G$ belongs to $N_{G}\left(M \times M^{b_{2}} \times \cdots \times M^{b_{m}}\right)$ if and only if

$$
\eta_{d}:=b_{d} x_{d} \tau_{d} b_{d+k}^{-1} \in N_{S_{n}}(M), \quad \forall d=1, \ldots, m .
$$

Moreover in this case

$$
\begin{gathered}
\eta:=\eta_{1} \eta_{1+k} \eta_{1+2 k} \cdots \eta_{1+(m-1) k}= \\
=x_{1} \tau_{1} x_{1+k} \tau_{1+k} \cdots x_{1+(m-1) k} \tau_{1+(m-1) k} \in N_{S_{n}}(M)-A_{n} .
\end{gathered}
$$

(2) Suppose $k>m$. For $d \in\{1, \ldots, m\}$ define $\tau_{d}$ to be $\tau$ if $d \leq 2 m-k$, and 1 if $d>2 m-k$. The element $\left(x_{1}, \ldots, x_{m}\right) \gamma^{k} \in G$ belongs to $N_{G}\left(M \times M^{b_{2}} \times\right.$ $\left.\cdots \times M^{b_{m}}\right)$ if and only if

$$
\eta_{d}:=b_{d} x_{d} \tau_{d} b_{d+k-m}^{-1} \in N_{S_{n}}(M), \quad \forall d=1, \ldots, m .
$$

Moreover in this case

$$
\begin{gathered}
\eta:=\eta_{1} \eta_{1+k-m} \eta_{1+2(k-m)} \cdots \eta_{1+(m-1)(k-m)}= \\
=x_{1} \tau_{1} x_{1+k-m} \tau_{1+k-m} \cdots x_{1+(m-1)(k-m)} \tau_{1+(m-1)(k-m)} \in N_{S_{n}}(M)-A_{n} .
\end{gathered}
$$

(3) If $N_{S_{n}}(M)$ contains $\eta$ (which depends only on $x_{1}, \ldots, x_{m}$ ), then there exist $a_{2}, \ldots, a_{m} \in A_{n}$ such that

$$
\left(x_{1}, \ldots, x_{m}\right) \gamma^{k} \in N_{G}\left(M \times M^{a_{2}} \times \cdots \times M^{a_{m}}\right) .
$$


Proof. Assume first that $k<m$. The element

$$
\left(x_{1}, \ldots, x_{m}\right) \gamma^{k}=\left(x_{1}, \ldots, x_{m-k}, x_{m-k+1} \tau, \ldots, x_{m} \tau\right) \delta^{k}
$$

belongs to $N_{G}\left(M \times M^{b_{2}} \times \cdots \times M^{b_{m}}\right)$ if and only if

$$
\begin{gathered}
\left(M^{x_{1}} \times M^{b_{2} x_{2}} \times \cdots \times M^{b_{m-k} x_{m-k}} \times M^{b_{m-k+1} x_{m-k+1} \tau} \times \cdots \times M^{b_{m} x_{m} \tau}\right)^{\delta^{k}}= \\
=M \times M^{b_{2}} \times \cdots \times M^{b_{m}},
\end{gathered}
$$

if and only if

$$
\begin{aligned}
M^{b_{m-k+1} x_{m-k+1} \tau} \times \cdots \times M^{b_{m} x_{m} \tau} \times M^{x_{1}} \times M^{b_{2} x_{2}} \times \cdots \times M^{b_{m-k} x_{m-k}}= \\
=M \times M^{b_{2}} \times \cdots \times M^{b_{m}} .
\end{aligned}
$$

In other words:

$$
\begin{gathered}
b_{m-k+1} x_{m-k+1} \tau, b_{m-k+2} x_{m-k+2} \tau b_{2}^{-1}, \ldots, b_{m} x_{m} \tau b_{k}^{-1}, \\
x_{1} b_{k+1}^{-1}, b_{2} x_{2} b_{k+2}^{-1}, \ldots, b_{m-k} x_{m-k} b_{m}^{-1} \in N_{S_{n}}(M) .
\end{gathered}
$$

For $d \in\{1, \ldots, m\}$ define $\tau_{d}$ to be $\tau$ if $d>m-k$, and 1 if $d \leq m-k$. The conditions we have are the following:

$$
\eta_{d}:=b_{d} x_{d} \tau_{d} b_{d+k}^{-1} \in N_{S_{n}}(M), \quad d=1, \ldots, m .
$$

Observe that since $k$ and $m$ are coprime,

$$
\left\{\tau_{1}, \tau_{1+k}, \tau_{1+2 k}, \ldots, \tau_{1+(m-1) k}\right\}=\left\{\tau_{1}, \ldots, \tau_{m}\right\} .
$$

Now

$$
\begin{gathered}
\eta:=\eta_{1} \eta_{1+k} \eta_{1+2 k} \cdots \eta_{1+(m-1) k}= \\
=x_{1} \tau_{1} x_{1+k} \tau_{1+k} \cdots x_{1+(m-1) k} \tau_{1+(m-1) k} \in N_{S_{n}}(M)
\end{gathered}
$$

is an odd element of $S_{n}$ since $\eta \equiv \tau^{k} \bmod \left(A_{n}\right)$ and $k$ is odd (being coprime to $2 m)$.

Point (2) follows easily from point (1) by noticing that $\left(\left(x_{1}, \ldots, x_{m}\right) \gamma^{k}\right)^{-1}=$ $\left(x_{1}^{-1}, \ldots, x_{m}^{-1}\right)^{\gamma^{k}} \gamma^{2 m-k}$.

Let us prove point (3). Suppose that the normalizer of $M$ in $S_{n}$ contains $\eta$. Assume that $k<m$ (the case $k>m$ is similar). For fixed elements $b_{2}, \ldots, b_{m} \in S_{n}$ define $\eta_{d}:=b_{d} x_{d} \tau_{d} b_{d+k}^{-1}$, for $d=1, \ldots, m$, and now choose $b_{2}, \ldots, b_{m}$ in such a way that $\eta_{1+k}, \eta_{1+2 k}, \ldots, \eta_{1+(m-1) k} \in N_{S_{n}}(M)$. Let $\eta_{1}$ be the element of $S_{n}$ such that $\eta_{1} \eta_{1+k} \cdots \eta_{1+(m-1) k}=\eta$. Then since $\eta \in N_{S_{n}}(M)$, also $\eta_{1} \in N_{S_{n}}(M)$. Now, a suitable power of $\left(x_{1}, \ldots, x_{m}\right) \gamma^{k}$ is of the form $\left(y_{1}, \ldots, y_{m}\right) \gamma$, with $y_{1}, \ldots, y_{m} \in A_{n}$. Since the element $\left(y_{1}, \ldots, y_{m}\right) \gamma \in G$ belongs to $N_{G}\left(M \times M^{b_{2}} \times \cdots \times M^{b_{m}}\right)$ we have

$$
b_{m} y_{m} \tau, y_{1} b_{2}^{-1}, b_{2} y_{2} b_{3}^{-1}, \ldots, b_{m-1} y_{m-1} b_{m}^{-1} \in N_{S_{n}}(M) .
$$

We may choose $a_{2}:=y_{1}, a_{3}:=y_{1} y_{2}, \ldots, a_{m}:=y_{1} y_{2} \cdots y_{m-1}$. In this way we get $M^{b_{i}}=M^{a_{i}}$ and $a_{i} \in A_{n}$, for $i=2, \ldots, m$.

From the proof of this proposition it easily follows that:

Corollary 2. If $M \leq A_{n}, b_{2}, \ldots, b_{m} \in \operatorname{Aut}\left(A_{n}\right)$ and $N_{G}\left(M \times M^{b_{2}} \times \cdots \times M^{b_{m}}\right)$ contains an element of the form $\left(x_{1}, \ldots, x_{m}\right) \gamma$ with $x_{1}, \ldots, x_{m} \in A_{n}$ then there exist $a_{2}, \ldots, a_{m} \in A_{n}$ such that $M^{b_{i}}=M^{a_{i}}$ for $i=2, \ldots, m$. 
Lemma 6. Let $r$ be a divisor of $m$, and let $x_{1}, \ldots, x_{m} \in A_{n}, a_{1}:=1, a_{2}, \ldots, a_{m} \in$ $S_{n}$. Let $M$ be a subgroup of $A_{n}$. The element $\left(x_{1}, \ldots, x_{m}\right) \gamma^{r} \in G$ belongs to $N_{G}\left(M \times M^{a_{2}} \times \cdots \times M^{a_{m}}\right)$ if and only if the following conditions are satisfied:

In particular

$$
\begin{gathered}
a_{m-r+i} x_{m-r+i} \tau a_{i}^{-1} \in N_{S_{n}}(M) \quad \forall i=1, \ldots, r ; \\
a_{i} x_{i} a_{r+i}^{-1} \in N_{S_{n}}(M) \quad \forall i=1, \ldots, m-r .
\end{gathered}
$$

$$
x_{i} x_{i+r} x_{i+2 r} \cdots x_{i+m-r} \tau \in N_{S_{n}}(M)^{a_{i}} \quad \forall i=1, \ldots, r .
$$

Now assume that $m$ is odd. Then the element $\left(x_{1}, \ldots, x_{m}\right) \gamma^{2} \in G$ belongs to $N_{G}\left(M \times M^{a_{2}} \times \cdots \times M^{a_{m}}\right)$ if and only if the following conditions are satisfied:

$$
\begin{gathered}
a_{m-1} x_{m-1} \tau, a_{m} x_{m} \tau a_{2}^{-1}, \\
x_{1} a_{3}^{-1}, a_{2} x_{2} a_{4}^{-1}, \ldots, a_{m-2} x_{m-2} a_{m}^{-1} \in N_{S_{n}}(M) .
\end{gathered}
$$

In particular

$$
x_{1} x_{3} \cdots x_{m} \tau x_{2} x_{4} \cdots x_{m-1} \tau \in N_{S_{n}}(M) .
$$

Proof. The element $\left(x_{1}, \ldots, x_{m}\right) \gamma^{r}=\left(x_{1}, \ldots, x_{m-r}, x_{m-r+1} \tau, \ldots, x_{m} \tau\right) \delta^{r}$ normalizes $M \times M^{a_{2}} \times \cdots \times M^{a_{m}}$ if and only if

$$
\left(M \times M^{a_{2}} \times \cdots \times M^{a_{m}}\right)^{\left(x_{1}, \ldots, x_{m-r}, x_{m-r+1} \tau, \ldots, x_{m} \tau\right) \delta^{r}}=M \times M^{a_{2}} \times \cdots \times M^{a_{m}},
$$

in other words

$$
\begin{gathered}
M^{a_{m-r+1} x_{m-r+1} \tau} \times \cdots \times M^{a_{m} x_{m} \tau} \times M^{x_{1}} \times M^{a_{2} x_{2}} \times \cdots \times M^{a_{m-r} x_{m-r}}= \\
=M \times M^{a_{2}} \times \cdots \times M^{a_{m}},
\end{gathered}
$$

and this leads to what is stated.

Now assume $m$ is odd. The element

$$
\left(x_{1}, \ldots, x_{m}\right) \gamma^{2}=\left(x_{1}, \ldots, x_{m-2}, x_{m-1} \tau, x_{m} \tau\right) \delta^{2}
$$

normalizes $M \times M^{a_{2}} \times \cdots \times M^{a_{m}}$ if and only if

$$
\left(M \times M^{a_{2}} \times \cdots \times M^{a_{m}}\right)^{\left(x_{1}, \ldots, x_{m-2}, x_{m-1} \tau, x_{m} \tau\right) \delta^{2}}=M \times M^{a_{2}} \times \cdots \times M^{a_{m}},
$$

in other words

$$
\begin{gathered}
M^{a_{m-1} x_{m-1} \tau} \times M^{a_{m} x_{m} \tau} \times M^{x_{1}} \times M^{a_{2} x_{2}} \times \cdots \times M^{a_{m-2} x_{m-2}}= \\
=M \times M^{a_{2}} \times \cdots \times M^{a_{m}},
\end{gathered}
$$

and this leads to what is stated.

Lemma 7. Let $r$ be a divisor of $m$. The element $\left(x_{1}, \ldots, x_{m}\right) \gamma$ normalizes

$$
\Delta:=\left\{\left(y_{1}, \ldots, y_{m / r}, y_{1}^{b_{21}}, \ldots, y_{m / r}^{b_{2, m / r}}, \ldots, y_{1}^{b_{r, 1}}, \ldots, y_{m / r}^{b_{r, m} / r}\right) \mid y_{1}, \ldots, y_{m / r} \in A_{n}\right\}
$$

if and only if (here $b_{1 i}=1$ for all $i=1, \ldots, m / r$ )

$$
b_{r, m / r} x_{m} \tau b_{i 1}=b_{i-1, m / r} x_{(i-1) m / r} \quad \forall i=2, \ldots, r
$$

and

$$
x_{j} b_{i, j+1}=b_{i, j} x_{(i-1)(m / r)+j} \quad \forall i=2, \ldots, r, j=1, \ldots, m / r-1 .
$$

In particular

$$
x_{1} \cdots x_{m} \tau=\left[x_{1} \cdots x_{m / r-1}\left(b_{r, m / r} x_{m} \tau\right)\right]^{r} .
$$

For $b \in S_{n}$ let $l_{r}(b)$ be the number of elements $s \in S_{n}$ such that $s^{r}=b$. Then

$$
\left|\left\{\left(x_{1}, \ldots, x_{m}\right) \gamma \in N_{G}(\Delta) \mid x_{1} \cdots x_{m} \tau=b\right\}\right|=l_{r}(b) \cdot\left|A_{n}\right|^{m / r-1} .
$$


In particular this number is 0 if $b \in A_{n}$ or if $r$ is even.

Proof. It is a direct computation. The element $\left(x_{1}, \ldots, x_{m}\right) \gamma$ belongs to $N_{G}(\Delta)$ if and only if for every $y_{1}, \ldots, y_{m / r} \in A_{n}$ the element

$$
\begin{gathered}
\left(y_{m / r}^{b_{r, m / r} x_{m} \tau}, y_{1}^{x_{1}}, \ldots, y_{m / r}^{x_{m / r}}, y_{1}^{b_{21} x_{m / r+1}}, \ldots, y_{m / r}^{b_{2, m / r} x_{2 m / r}}, \ldots,\right. \\
\left.y_{1}^{b_{r, 1} x_{(r-1) m / r+1}}, \ldots, y_{m / r-1}^{b_{r, m / r-1} x_{m-1}}\right)
\end{gathered}
$$

belongs to $\Delta$, and this leads to the stated conditions.

Using these conditions we see that for every $1 \leq i \leq r-1$,

$$
\begin{gathered}
x_{1} \cdots x_{m / r-1} b_{r, m / r} x_{m} \tau= \\
=b_{i, 1} x_{(i-1) m / r+1} x_{(i-1) m / r+2} \cdots x_{(i-1) m / r+m / r-1} x_{i m / r} b_{i+1,1}^{-1},
\end{gathered}
$$

and

$$
x_{1} \cdots x_{m / r-1} b_{r, m / r} x_{m} \tau=b_{r, 1} x_{(r-1) m / r+1} \cdots x_{m-1} x_{m} \tau .
$$

It follows that

$$
\left(x_{1} \cdots x_{m / r-1} b_{r, m / r} x_{m} \tau\right)^{r}=x_{1} \cdots x_{m} \tau .
$$

The last two statements follow easily from the first two.

\section{Proof of Theorem 1}

In this section we prove Theorem 1 for $m \geq 2$ (the case $m=1$ is proved in [12]).

The next definition was introduced in [12].

Definition 1 (Definite unbeatability). Let $X$ be a finite group. Let $\mathcal{H}$ be a set of proper subgroups of $X$, and let $\Pi \subseteq X$. Suppose that the following four conditions hold on $\mathcal{H}$ and $\Pi$.

(1) $\Pi \cap H \neq \emptyset$ for every $H \in \mathcal{H}$;

(2) $\Pi \subseteq \bigcup_{H \in \mathcal{H}} H$;

(3) $\Pi \cap H_{1} \cap H_{2}=\emptyset$ for every distinct pair of subgroups $H_{1}$ and $H_{2}$ of $\mathcal{H}$;

(4) $|\Pi \cap K| \leq|\Pi \cap H|$ for every $H \in \mathcal{H}$ and $K<X$ with $K \notin \mathcal{H}$.

Then $\mathcal{H}$ is said to be definitely unbeatable on $\Pi$.

For $\Pi \subseteq X$ let $\sigma_{X}(\Pi)$ be the least cardinality of a family of proper subgroups of $X$ whose union contains $\Pi$. The next lemma is straightforward so we state it without proof.

Lemma 8. If $\mathcal{H}$ is definitely unbeatable on $\Pi$ then $\sigma_{X}(\Pi)=|\mathcal{H}|$.

It follows that if $\mathcal{H}$ is definitely unbeatable on $\Pi$ then $|\mathcal{H}|=\sigma_{X}(\Pi) \leq \sigma(X)$.

Let us fix the notations.

Notations 1. Let $n, m$ be positive integers, with $m \geq 2$ and $n \geq 5$. Let $A, B$ be two fixed subsets of $S_{n}-A_{n}$, and let $C$ be a fixed subset of $A_{n}$. For a prime divisor $r$ of $m$ define $\Omega_{r}$ to be the set

$\left\{\left(x_{1}, \ldots, x_{m}\right) \gamma^{r} \mid x_{1} x_{1+r} x_{1+2 r} \cdots x_{1+m-r} \tau \in A, x_{2} x_{2+r} x_{2+2 r} \cdots x_{2+m-r} \tau \in B\right\}$.

If $m$ is odd let

$$
\Omega_{2}:=\left\{\left(x_{1}, \ldots, x_{m}\right) \gamma^{2} \mid x_{1} x_{3} \cdots x_{m} \tau x_{2} x_{4} \cdots x_{m-1} \tau \in C\right\} .
$$

For a prime divisor $r$ of $2 m$ let $H_{r}$ be the pre-image of $\left\langle\gamma^{r}\right\rangle$ via the projection $G \rightarrow\langle\gamma\rangle$. Let $\Pi$ be a fixed subset of $S_{n}-A_{n}$, and let

$$
\Omega_{1}:=\left\{\left(x_{1}, \ldots, x_{m}\right) \gamma \mid x_{1} \cdots x_{m} \tau \in \Pi\right\} .
$$


Assume that $n \geq 5$ is odd. Let $K_{1}, \ldots, K_{t}$ be the intransitive maximal subgroups of $A_{n}$. Let $\Sigma$ be the subset of $S_{n}$ consisting of the $(k, n-k)$-cycles where $1 \leq k \leq$ $n-1$, and let $\Pi$ be a fixed subset of $\Sigma$. Call $I:=\left\{i \in\{1, \ldots, t\} \mid N_{S_{n}}\left(K_{i}\right) \cap \Pi \neq \emptyset\right\}$. Let

$$
\mathcal{L}:=\left\{N_{G}\left(K_{i} \times K_{i}^{a_{2}} \times \cdots \times K_{i}^{a_{m}}\right) \mid i \in I, a_{2}, \ldots, a_{m} \in A_{n}\right\} .
$$

Let $A$ be the set of the $(2, n-2)$-cycles of $S_{n}$, let $B$ be the set of the $(n-1)$-cycles of $S_{n}$, for $m$ odd let $C$ be:

- the set of the $n$-cycles of $S_{n}$ if either $n \geq 7$, or $n=5$ and $m \notin\{5,7\}$;

- a subset of $S_{5}$ consisting of 125 -cycles, two in each Sylow 5-subgroup, if $n=5$ and $m \in\{5,7\}$.

If $m$ is even or $(n, m)=(5,3)$ let $C=\emptyset$. We have $|A|=\left|A_{n}\right| /(n-2),|B|=$ $2\left|A_{n}\right| /(n-1),|C|=2\left|A_{n}\right| / n$ if $n \geq 7$ or $n=5, m \notin\{3,5,7\},|C|=12$ if $n=5$, $m \in\{5,7\}$, and $\left|\Omega_{r}\right|=\frac{2}{(n-1)(n-2)}\left|A_{n}\right|^{m}$ if $r \neq 2$ or $m$ is even, while if $r=2$ and $m$ is odd then $\left|\Omega_{r}\right|=(2 / n)\left|A_{n}\right|^{m}$. Suppose we are in one of the following cases:

(1) $n=5$ and $\Pi=\{(2354)$, , (4521), (4132), (1253), (4531), (3245), (1352), (2314), (4125), (3541)\};

(2) $n \geq 7$ and $\Pi=\Sigma$.

Let $r_{1}, \ldots, r_{\omega(2 m)}$ be the distinct prime factors of $2 m$. By Lemma 5 and Corollary 2 the family $\mathcal{H}:=\mathcal{L} \cup\left\{H_{r_{1}}, \ldots, H_{r_{\omega(2 m)}}\right\}$ covers $G$ if $n \neq 5$. In fact the odd elements of $S_{n}$ are covered by the intransitive maximal subgroups of $S_{n}$.

Proposition 2. With the notations and assumptions above, we have:

(1) If $(n, m) \neq(5,3)$, $\mathcal{H}$ is definitely unbeatable on $\Omega:=\Omega_{1} \cup \Omega_{r_{1}} \cup \cdots \cup \Omega_{r_{\omega(2 m)}}$.

(2) $\mathcal{L}$ is definitely unbeatable on $\Omega_{1}$.

Proof. We will verify the four conditions of Definition 1 for both $\mathcal{H}$ and $\mathcal{L}$. Lemmas 4 and 6 imply that if $H$ is a maximal subgroup of $G$ of product type and $r$ is a prime divisor of $m$ then $H \cap \Omega_{r}=\emptyset$; in particular $H \cap \Omega=H \cap\left(\Omega_{1} \cup \Omega_{2}\right)$. If $H \in \mathcal{L}$ then $H \cap \Omega_{2}=\emptyset$. Moreover $\Omega_{r} \subset H_{r}$ for every prime divisor $r$ of $2 m$ and $H_{r} \cap H_{s} \cap \Omega=\emptyset$ for every two distinct prime divisors $r, s$ of $2 m$. All this implies that the first three conditions of Definition 1 hold for $\mathcal{H}$ if they hold for $\mathcal{L}$. We will check them now.

Recall first that if $K$ is a subgroup of $A_{n}$ and $x_{1}, \ldots, x_{m}, a_{2}, \ldots, a_{m} \in A_{n}$ then $\left(x_{1}, \ldots, x_{m}\right) \gamma \in N_{G}\left(K \times K^{a_{2}} \times \cdots \times K^{a_{m}}\right)$ if and only if $a_{m} x_{m} \tau \in N_{S_{n}}(K), x_{1} \in K a_{2}, x_{2} \in a_{2}^{-1} K a_{3}, \cdots, x_{m-1} \in a_{m-1}^{-1} K a_{m}$.

(1) We show that $\Omega_{1} \cap H \neq \emptyset$ for every $H=N_{G}\left(K_{i} \times K_{i}^{a_{2}} \times \cdots \times K_{i}^{a_{m}}\right) \in \mathcal{L}$. Choose the element $\left(x_{1}, \ldots, x_{m}\right) \gamma$ in the intersection in this way: $x_{1}=a_{2}$, $x_{2}=a_{2}^{-1} a_{3}, \ldots, x_{m-1}=a_{m-1}^{-1} a_{m}$ and $x_{m}$ such that $x_{1} \cdots x_{m} \tau \in \Pi \cap$ $N_{S_{n}}\left(K_{i}\right)$.

(2) We show that $\Omega_{1} \subseteq \bigcup_{H \in \mathcal{L}} H$. Given $\left(x_{1}, \ldots, x_{m}\right) \gamma \in \Omega_{1}$ choose $i \in I$ such that $x_{1} \cdots x_{m} \tau \in N_{S_{n}}\left(K_{i}\right)$ and $a_{2}=x_{1}, a_{3}=x_{1} x_{2}, \ldots, a_{m}=$ $x_{1} x_{2} \cdots x_{m-1}$. Then choose $H:=N_{G}\left(K_{i} \times K_{i}^{a_{2}} \times \cdots \times K_{i}^{a_{m}}\right)$.

(3) We show that $\Omega_{1} \cap N_{G}\left(K_{i} \times K_{i}^{a_{2}} \times \cdots \times K_{i}^{a_{m}}\right) \cap N_{G}\left(K_{j} \times K_{j}^{b_{2}} \times \cdots \times K_{j}^{b_{m}}\right)=\emptyset$ for $N_{G}\left(K_{i} \times K_{i}^{a_{2}} \times \cdots \times K_{i}^{a_{m}}\right) \neq N_{G}\left(K_{j} \times K_{j}^{b_{2}} \times \cdots \times K_{j}^{b_{m}}\right)$ belonging to $\mathcal{L}$. If $\left(x_{1}, \ldots, x_{m}\right) \gamma$ belongs to the stated intersection then $x_{1} \cdots x_{m} \tau \in$ $N_{S_{n}}\left(K_{i}\right) \cap N_{S_{n}}\left(K_{j}\right) \cap \Pi$ with $i \neq j$ (which is impossible) or $i=j$ and

$$
x_{k} \in a_{k}^{-1} K_{i} a_{k+1} \cap b_{k}^{-1} K_{i} b_{k+1}
$$


for $k=1, \ldots, m$, where $a_{1}:=1$. This easily implies that $K_{i}^{a_{k}}=K_{i}^{b_{k}}$ for $k=2, \ldots, m$, contradiction.

We now prove that $|H \cap \Omega| \geq\left|H^{\prime} \cap \Omega\right|$ for every $H \in \mathcal{H}, H^{\prime}$ maximal subgroup of $G$ with $H^{\prime} \notin \mathcal{H}$. Note that this indeed proves condition (4) of Definition 1 for both $\mathcal{H}$ and $\mathcal{L}$ since for every prime divisor $r$ of $2 m$ and every $H \in \mathcal{L}$ we have $H_{r} \cap \Omega_{1}=\emptyset$ and $H \cap \Omega_{r}=\emptyset$.

First we prove that if $K \notin\left\{K_{i} \mid i \in I\right\}$ is a subgroup of $A_{n}$ of the form $R \cap A_{n}$ where $R$ is a maximal subgroup of $S_{n}$ (cfr. section 1) then

$$
\left|\Omega \cap N_{G}\left(K_{i} \times K_{i}^{a_{2}} \times \cdots \times K_{i}^{a_{m}}\right)\right| \geq\left|\Omega \cap N_{G}\left(K \times K^{b_{2}} \times \cdots \times K^{b_{m}}\right)\right| .
$$

Notice that since the right hand side of this inequality is zero if $K$ is intransitive (this can happen if $n=5$ ), we may assume that $K$ is transitive. As we have already noticed this inequality re-writes as

$$
\left|\Omega_{1} \cap N_{G}\left(K_{i} \times K_{i}^{a_{2}} \times \cdots \times K_{i}^{a_{m}}\right)\right| \geq\left|\left(\Omega_{1} \cup \Omega_{2}\right) \cap N_{G}\left(K \times K^{b_{2}} \times \cdots \times K^{b_{m}}\right)\right| .
$$

The size of $\Omega_{1} \cap N_{G}\left(L \times L^{a_{2}} \times \cdots \times L^{a_{m}}\right)$ in general (for a subgroup $L$ of $A_{n}$ and some $\left.a_{2}, \ldots, a_{m} \in S_{n}\right)$ is $|L|^{m-1} \cdot\left|N_{S_{n}}(L) \cap \Pi\right|$, and if $m$ is odd the size of $\Omega_{2} \cap N_{G}\left(L \times L^{a_{2}} \times \cdots \times L^{a_{m}}\right)$ is $|L|^{m-1} \cdot|L \cap C|$. Therefore we have to show that

$$
\left|K_{i}\right|^{m-1} \cdot\left|N_{S_{n}}\left(K_{i}\right) \cap \Pi\right| \geq|K|^{m-1} \cdot\left|N_{S_{n}}(K) \cap(\Pi \cup C)\right| .
$$

- Suppose $n=5$. The transitive maximal subgroups of $A_{5}$ have order 10 . Moreover the only intransitive maximal subgroups of $A_{5}$ whose normalizers in $S_{5}$ intersect $\Pi$ are the five point stabilizers. If $m$ is even or $m=3$ then $C=\emptyset$ and $\left|N_{S_{5}}\left(K_{i}\right) \cap \Pi\right|=\left|N_{S_{5}}(K) \cap \Pi\right|=2$ for every $i \in I$, thus (*) is true. If $m \notin\{5,7\}$ is odd then $\left|N_{S_{n}}(K) \cap(\Pi \cup C)\right|=6$ and $(*)$ becomes $12^{m-1} \cdot 2 \geq 10^{m-1} \cdot 6$, which is true for $m \geq 8$. If $m \in\{5,7\}$ then $\left|N_{S_{n}}(K) \cap(\Pi \cup C)\right|=4$ and $(*)$ becomes $12^{m-1} \cdot 2 \geq 10^{m-1} \cdot 4$, which is true.

- Suppose $n=7$. The left hand side is at least $72^{m-1} \cdot 12$. Since the transitive maximal subgroups of $S_{7}$ different from $A_{7}$ have size 42 and contain 20 elements of $\Pi \cup C$, it suffices to show that $72^{m-1} \cdot 12 \geq 21^{m-1} \cdot 20$, i.e. $(72 / 21)^{m} \geq 40 / 7$, which is true for $m \geq 2$.

- Suppose $n=9$. The smallest maximal intransitive subgroup of $A_{9}$ is the one of type $(4,5)$, it has size 1440 and the size of the intersection of its normalizer in $S_{9}$ with $\Pi$ is the smallest possible, $3 ! \cdot 4 !=144$. Thus the left hand side of $(*)$ is at least $1440^{m-1} \cdot 144$. The right hand side is at most $\max \left(216^{m-1} \cdot 72,648^{m-1} \cdot 432\right)$ (note that the maximal subgroups of $A_{9}$ isomorphic to $\operatorname{Aut}(P S L(2,8))$ are not of the form $R \cap A_{9}$ with $R$ maximal in $S_{9}$ : cfr. section (1). Therefore it suffices to show that $1440^{m-1} \cdot 144 \geq$ $648^{m-1} \cdot 432$, and this is true for $m \geq 3$. If $m=2$ then $C=\emptyset$ and it suffices to show that $1440 \cdot 144 \geq 648 \cdot 288$ (recall that the imprimitive maximal subgroups of $S_{9}$ contain 1449 -cycles and $288(6,3)$-cycles), which is true.

- Suppose $n \geq 11$. Then $\left|K_{i}\right| \geq|K|$ by Corollary 1, and the inequality $\left|N_{S_{n}}\left(K_{i}\right) \cap \Pi\right| \geq\left|N_{S_{n}}(K) \cap(\Pi \cup C)\right|$ is proved in claim 3.2 of [12].

Now we prove that if $N_{G}(\Delta)$ is a maximal subgroup of $G$ of diagonal type (its existence implies that $m$ is not a power of 2 by Lemma (7) and $i \in\{1, \ldots, t\}$, $a_{2}, \ldots, a_{m} \in A_{n}$ then

$$
\left|\Omega \cap N_{G}\left(K_{i} \times K_{i}^{a_{2}} \times \cdots \times K_{i}^{a_{m}}\right)\right| \geq\left|\Omega \cap N_{G}(\Delta)\right| .
$$


The right hand side is at most $\left|N_{G}(\Delta)\right| \leq 2 m\left|A_{n}\right|^{m / p}$, where $p$ is the smallest prime divisor of $m$, hence we are reduced to prove that $\left|K_{i}\right|^{m-1} \cdot\left|N_{S_{n}}\left(K_{i}\right) \cap \Pi\right| \geq$ $2 m\left|A_{n}\right|^{m / p}$. Since if $K_{i}$ is of type $(k, n-k)$ then $\left|N_{S_{n}}\left(K_{i}\right) \cap \Pi\right|=(k-1) !(n-k-1)$ !, we obtain $(2 /(k(n-k)))\left|K_{i}\right|^{m} \geq 2 m\left|A_{n}\right|^{m / p}$. Since $k(n-k) \leq((n-1) / 2)((n+1) / 2)$, it suffices to show that

$$
\frac{8}{n^{2}-1}\left|K_{i}\right|^{m} \geq 2 m \cdot\left|A_{n}\right|^{m / p} .
$$

Note that if $s$ is a divisor of $m$ and $L_{s}$ denotes the set of elements of $G$ of the form $\left(x_{1}, \ldots, x_{m}\right) \gamma^{s}$ then $\left|N_{G}(\Delta) \cap L_{s}\right|=|\Delta|$. Therefore by Lemma 7 if $N_{G}(\Delta)$ is of diagonal type 2 then it suffices to show that

$$
\frac{8}{n^{2}-1}\left|K_{i}\right|^{m} \geq \omega(m) \cdot\left|A_{n}\right|^{m / 2}
$$

- If $n=5$ then $\left|K_{i}\right| \geq \frac{1}{2} 2 ! 3 !=6$ and (1) is true for $m \geq 3$. If $n=9$ then $\left|K_{i}\right| \geq \frac{1}{2} 4 ! 5 !=1440$ and $(1)$ is true for $m \geq 3$. If $n=11$ then $\left|K_{i}\right| \geq \frac{1}{2} 5 ! 6 !=$ 43200 and ( 1 ) is true for $m \geq 2$. If $n=13$ then $\left|K_{i}\right| \geq \frac{1}{2} 6 ! 7 !=1814400$ and (1) is true for $m \geq 2$.

- Suppose $n=7$. Then $\left|K_{i}\right| \geq 72$, thus it suffices to show (1): $72^{m} \geq$ $12 m \cdot 2520^{m / 2}$, i.e. $(72 / \sqrt{2520})^{m} / m \geq 12$. This is true for $m \geq 15$. If $p \geq 3$ it suffices to show that $(72 / \sqrt[3]{2520})^{m} / m \geq 12$, which is true for $m \geq 3$. Thus we are done if $p$ is odd. If $m \in\{10,12,14\}$ then $\omega(m)=2$ and using (2) we are reduced to show that $(72 / \sqrt{2520})^{m} \geq 12$, which is true.

We are left with the case $m=6$. It is easy to see that in general if $H$ is a maximal subgroup of $G$ of diagonal type 2 and $r$ is a prime divisor of $m$ then $\left|\Omega_{r} \cap H\right| \leq\left|A_{n}\right|^{m / 2-1} \cdot \min (|A|,|B|)$ (just use the definition of $\Omega_{r}$ ). In our case $\min (|A|,|B|)=|A|=504$, and $72^{6} \geq \omega(6) \cdot 6 \cdot 2520^{2} \cdot 504$.

- Suppose $n \geq 15$. Then $\left|K_{i}\right|^{3 / 2} \geq\left|A_{n}\right|$ by Lemma 3. so using (1) we are reduced to prove that $\left(8 /\left(n^{2}-1\right)\right)\left|A_{n}\right|^{\frac{2}{3} m} \geq 2 m\left|A_{n}\right|^{m / 2}$, i.e. $\left|A_{n}\right|^{m / 6} \geq$ $(m / 4)\left(n^{2}-1\right)$. This is clearly true for every $m$ since $n \geq 15$.

Now we prove that if $(n, m) \neq(5,3)$ then $\left|H_{r} \cap \Omega\right| \geq|H \cap \Omega|$ for every maximal subgroup $H$ of $G$ of product type out of $\mathcal{H}$ and for every prime divisor $r$ of $2 \mathrm{~m}$. Let $L$ be the transitive subgroup of $A_{n}$ such that $H=N_{G}\left(L \times L^{a_{2}} \times \cdots \times L^{a_{m}}\right)$. Note that

$$
|H \cap \Omega|=\left|H \cap\left(\Omega_{1} \cup \Omega_{2}\right)\right|=|L|^{m-1} \cdot\left(\left|N_{S_{n}}(L) \cap \Pi\right|+|L \cap C|\right) .
$$

Suppose first that $r \neq 2$ or $m$ is even. All we have to prove is that

$$
\begin{gathered}
\frac{2}{(n-1)(n-2)}\left|A_{n}\right|^{m}=\left|\Omega_{r}\right|=\left|H_{r} \cap \Omega\right| \geq\left|H \cap\left(\Omega_{1} \cup \Omega_{2}\right)\right|= \\
=|L|^{m-1} \cdot\left(\left|N_{S_{n}}(L) \cap \Pi\right|+|L \cap C|\right) .
\end{gathered}
$$

This is easily seen to be true for $n \in\{5,7,9\}$. Suppose $n \geq 11$. It suffices to show that $\frac{2}{(n-1)(n-2)}\left|A_{n}\right|^{m} \geq 2|R|^{m}$ for any maximal transitive $\operatorname{subgroup} R$ of $S_{n}$ different from $A_{n}$, i.e. $\left(\left|S_{n}: R\right| / 2\right)^{m} \geq(n-1)(n-2)$, and this is true by Corollary 1. being true for $m=1:\left|S_{n}: R\right| / 2 \geq\left(\begin{array}{l}n \\ 5\end{array}\right) / 2>(n-1)(n-2)$ since $n>8$.

Assume now that $r=2$ and $m$ is odd. All we have to prove is that

$$
\begin{gathered}
|C| \cdot\left|A_{n}\right|^{m-1}=\left|\Omega_{2}\right|=\left|H_{2} \cap \Omega\right| \geq\left|H \cap\left(\Omega_{1} \cup \Omega_{2}\right)\right|= \\
=|L|^{m-1} \cdot\left(\left|N_{S_{n}}(L) \cap \Pi\right|+|L \cap C|\right) .
\end{gathered}
$$


It suffices to prove that for every transitive subgroup $R$ of $S_{n}$ not containing $A_{n}$ we have $|C| \cdot\left|A_{n}\right|^{m-1} \geq 2|R|^{m}$, i.e. $\left(\left|S_{n}: R\right| / 2\right)^{m} \geq\left|S_{n}\right| /|C|$. If $n>5$ this follows from $\left|S_{n}: R\right| \geq n$, if $n=5$ this follows from $|C| \geq 12$.

Now we prove that if $(n, m) \neq(5,3)$ then $\left|H_{r} \cap \Omega\right| \geq|H \cap \Omega|$ for every prime divisor $r$ of $2 m$ and every maximal subgroup $H$ of $G$ of diagonal type. Notice that $|H| \leq 2 m\left|A_{n}\right|^{m / 2}$, hence if $r \neq 2$ or $m$ is even we are reduced to prove that $2\left|A_{n}\right|^{m} /((n-1)(n-2)) \geq 2 m\left|A_{n}\right|^{m / 2}$, and this is clearly true for every $m$ and $n \geq 5$. If $r=2$ and $m$ is odd we have to prove that $(2 / n)\left|A_{n}\right|^{m} \geq 2 m\left|A_{n}\right|^{m / 2}$, and this is clearly true for every $m$ and $n \geq 5$.

Note that Proposition 2 implies Theorem 1 if $n>5$ is odd.

Observation 1. Let $\mathcal{K}$ be a minimal cover of the finite group $X$, so that $|\mathcal{K}|=$ $\sigma(X)$, and let $\mathcal{K}_{1}$ be a subset of $\mathcal{K}$. Let $\Omega$ be a subset of $X-\bigcup_{K \in \mathcal{K}_{1}} K$. Then $\left|\mathcal{K}_{1}\right|+\sigma_{X}(\Omega) \leq \sigma(X)$, where $\sigma_{X}(\Omega)$ denotes the least number of proper subgroups of $X$ needed to cover $\Omega$.

Suppose that $n=5$ and all the prime divisors of $m$ belong to $\{2,3\}$. Fix a minimal cover $\mathcal{K}$ of $G$. Let $\mathcal{K}_{0}$ be the family of the maximal subgroups of $G$ of the form $N_{G}\left(M \times M^{a_{2}} \times \cdots \times M^{a_{m}}\right)$ with $a_{2}, \ldots, a_{m} \in A_{5}$ and $M$ an intransitive maximal subgroup of $A_{5}$ of type $(3,2)$. Since the $(3,2)$-cycles are not of the form $x^{2}$ or $x^{3}$ for $x \in S_{5}$, by Lemma 7 the only maximal subgroups of $G$ which contain elements of the form $\left(x_{1}, \ldots, x_{m}\right) \gamma$ where $x_{1} \cdots x_{m} \tau$ is a $(3,2)$-cycle are the subgroups in $\mathcal{K}_{0}$. In particular $\mathcal{K}_{0} \subset \mathcal{K}$. In the following we use Notations 1, with $A$ the set of the $(3,2)$-cycles, $B$ the set of the 4 -cycles and $C$ the set of the 5 -cycles.

Suppose that $m$ is even, and let $\mathcal{K}_{1}:=\mathcal{K}_{0}$. For every $K \in \mathcal{K}_{1}$ we have $\Omega_{1} \cap K=$ $\Omega_{2} \cap K=\Omega_{3} \cap K=\emptyset$, thus by Observation 1 and Proposition $2\left|\mathcal{K}_{1}\right|+|\mathcal{H}| \leq \sigma(G)$, and we have equality since $\mathcal{K}_{1} \cup \mathcal{H}$ covers $G$.

Suppose that $m$ is a power of 3 , and let $\mathcal{K}_{1}:=\mathcal{K}_{0} \cup\left\{H_{2}, H_{3}\right\}$. If either $H_{2} \notin \mathcal{K}$ or $H_{3} \notin \mathcal{K}$ then in order to cover $\Omega_{2} \cup \Omega_{3}$ we need at least

$$
\frac{\min \left\{\left|\Omega_{2}\right|,\left|\Omega_{3}\right|\right\}}{\left|N_{G}(\Delta)\right|}=\frac{|A| \cdot|B| \cdot\left|A_{5}\right|^{m-1}}{2 m \cdot\left|A_{5}\right|^{m / 3}}=(5 / m) \cdot 60^{2 m / 3-1}
$$

subgroups, where $N_{G}(\Delta)$ is a maximal subgroup of $G$ of diagonal type. Since $\sigma(G) \leq 2+5^{m}+10^{m}$, we obtain that $10^{m}+(5 / m) 60^{2 m / 3-1} \leq 2+5^{m}+10^{m}$, contradiction. Therefore $\mathcal{K}_{1} \subset \mathcal{K}$. Since $\Omega_{1} \cap K=\emptyset$ for every $K \in \mathcal{K}_{1}$, by Observation 1 and Proposition 2 we obtain that $2+5^{m}+10^{m} \leq \sigma(G)$, thus we have equality.

Assume now that $n$ is any positive integer at least 5 . The following observation follows easily from the proof of Proposition 2

Observation 2. Let $\mathcal{A}$ be a family of proper subgroups of $A_{n}$, and let

$$
\mathcal{K}:=\left\{N_{G}\left(M \times M^{a_{2}} \times \cdots \times M^{a_{m}}\right) \mid a_{2}, \ldots, a_{m} \in A_{n}, M \in \mathcal{A}\right\} .
$$

Let $\Pi$ be a subset of $S_{n}$ such that $\mathcal{A}$ is definitely unbeatable on $\Pi$. Let

$$
\Omega:=\left\{\left(x_{1}, \ldots, x_{m}\right) \gamma \in G \mid x_{1} \cdots x_{m} \tau \in \Pi\right\} .
$$

Suppose that the following two conditions hold:

(1) $|M| \geq|K|$ for every $M \in \mathcal{A}$ and every maximal subgroup $K$ of $A_{n}$ such that $N_{S_{n}}(K) \cap \Pi \neq \emptyset$. 
(2) $|M|^{m-1} \cdot\left|N_{S_{n}}(M) \cap \Pi\right| \geq|H \cap \Omega|$ for every $M \in \mathcal{A}$ and every maximal subgroup $H$ of $G$ of diagonal type. Note that this is true if

$$
|M|^{m-1} \cdot\left|N_{S_{n}}(M) \cap \Pi\right| \geq 2 m\left|A_{n}\right|^{m / p}
$$

where $p$ is the smallest prime divisor of $m$ such that there exists a maximal subgroup of $G$ of diagonal type $p$ whose intersection with $\Omega$ is non-empty.

Then the family $\mathcal{K}$ of subgroups of $G$ is definitely unbeatable on $\Omega$. In particular $|\mathcal{K}| \leq \sigma(G)$.

Let us apply this observation to the cases we are left with.

Let $n=5$. Let $\mathcal{A}$ be the set of the intransitive maximal subgroups of $A_{5}$ of type $(3,2)$ and let $\Pi$ be the set of the $(3,2)$-cycles in $S_{5}$. Condition (1) of Observation 2 is clearly verified. Let us prove condition (2). By Lemma 7 we may assume $p \geq 5$ (the elements of $\Pi$ have no square roots nor cubic roots in $S_{5}$ ). The inequality $6^{m-1} \cdot 2 \geq 2 m \cdot 60^{m / p}$ is then true. We obtain $\sigma(G) \geq 10^{m}$.

Let $n=6$. Fix a minimal cover $\mathcal{M}$ of $G$ consisting of maximal subgroups. Let $\mathcal{K}_{0}$ be the family of the maximal subgroups of $G$ of the form $N_{G}\left(M \times M^{a_{2}} \times \cdots \times M^{a_{m}}\right)$ where $M$ is a subgroup of $A_{6}$ isomorphic to $A_{5}$, so that $\left|\mathcal{K}_{0}\right|=12 \cdot 6^{m-1}$. Let us use Notations 1. Let $\mathcal{K}_{1}$ be the set consisting of the subgroups in $\mathcal{K}_{0}$ and the subgroups $H_{r}$ for $r$ a prime divisor of $m$. Since $S_{6}-A_{6}$ is covered by the two conjugacy classes of maximal subgroups of $S_{6}$ isomorphic to $S_{5}, \mathcal{K}_{1} \cup\left\{H_{2}\right\}$ covers $G$, in particular $\sigma(G) \leq \omega(2 m)+2 \cdot 6^{m}$. It is easy to see that $H \cong A_{5}^{m} \rtimes C_{2 m}$ for every $H \in \mathcal{K}_{0}$, therefore

$$
\sigma(H) \geq 10^{m}>\omega(2 m)+2 \cdot 6^{m} \geq \sigma(G) .
$$

By Lemma 1 in $\left[7\right.$ we deduce that $\mathcal{K}_{0} \subset \mathcal{M}$. Let $A$ be the set of the $(3,2)$-cycles in $S_{6}$, let $B$ be the set of the 6 -cycles in $S_{6}$, and let $C$ be the set of the 3-cycles in $S_{6}$. Since no subgroup of $S_{6}$ intersects both $A$ and $B, H \cap \Omega_{r}=\emptyset$ for every prime divisor $r$ of $m$ and every maximal subgroup $H$ of $G$ of product type. If $H$ is a maximal subgroup of $G$ of diagonal type (in particular $m$ is not a power of 2 by Lemma 77) then $\left|H \cap \Omega_{r}\right| \leq|H \cap \operatorname{soc}(G)|$. Therefore if $r$ is a prime divisor of $m$ and $H_{r} \notin \mathcal{M}$ then in order to cover $\Omega_{r}$ we need at least

$$
\frac{\min _{r}\left|\Omega_{r}\right|}{|H \cap \operatorname{soc}(G)|} \geq \frac{40 \cdot 360^{m-1}}{360^{m / 2}}=40 \cdot 360^{m / 2-1}
$$

subgroups. Since $m \geq 3$, this contradicts $\sigma(G) \leq \omega(2 m)+2 \cdot 6^{m}$. Therefore $\mathcal{K}_{1} \subseteq \mathcal{M}$. If $m$ is even then $\mathcal{K}_{1}$ covers $G$, thus $\mathcal{K}_{1}=\mathcal{M}$ and we are done. Suppose $m$ is odd. Since the subgroups of $S_{6}$ isomorphic to $S_{5}$ do not intersect $C$, the family $\mathcal{K}_{1}$ does not cover $\Omega_{2}$. Since $\Omega_{2} \subset H_{2}$ and $\mathcal{K}_{1} \cup\left\{H_{2}\right\}$ covers $G$, we obtain $\sigma(G)=|\mathcal{M}|=\omega(2 m)+2 \cdot 6^{m}$.

Let $n \geq 8$ be even. Let $\Pi$ be the set of the $n$-cycles in $S_{n}$, and let $\mathcal{A}$ be the family of the maximal imprimitive subgroups of $A_{n}$ corresponding to the partitions given by two subsets of $\{1, \ldots, n\}$ of size $n / 2$. In [12] (claims 3.3 and 3.4) it is proved that if $n \geq 8$ then $\mathcal{A}$ is definitely unbeatable on $\Pi$. Condition (1) of Observation 2 follows from Lemma 1 and the fact that the order of a primitive maximal subgroup of $A_{n}$ is at most $2.6^{n}$ (see [11). In fact $(n / 2) !^{2} \geq 2.6^{n}$ if $n \geq 10$, and all the maximal subgroups of $A_{8}$ whose normalizers in $S_{8}$ contain 8-cycles belong to $\mathcal{A}$. We now prove condition (2). We may assume that $m$ is not a power of 2 by Lemma 7. If $n \in\{8,10\}$ then $|K|^{m-1} \cdot\left|N_{S_{n}}(K) \cap \Pi\right| \geq 2 m\left|A_{n}\right|^{m / 2}$ whenever $K \in \mathcal{A}$. Suppose $n \geq 12$. Using Lemma 3 we see that $|K|^{3 / 2} \geq\left|A_{n}\right|$ for every $K \in \mathcal{A}$. 
Therefore since $m \geq 2$ is not a power of 2 , if $p$ is the smallest prime divisor of $m$ then $|K|^{m-1} \geq\left|A_{n}\right|^{(2 / 3)(m-1)} \geq 2 m\left|A_{n}\right|^{m / p}$ for $K \in \mathcal{A}$ (if $m \geq 5$ this follows from $p \geq 2$ ). Applying Observation 2 we obtain that

$$
\left(\frac{1}{2}\left(\begin{array}{c}
n \\
n / 2
\end{array}\right)\right)^{m} \leq \sigma(G) \leq \omega(2 m)+\left(\frac{1}{2}\left(\begin{array}{c}
n \\
n / 2
\end{array}\right)\right)^{m}+\sum_{i=1}^{[n / 3]}\left(\begin{array}{c}
n \\
i
\end{array}\right)^{m} .
$$

The upper bound is obtained by observing that the non- $n$-cycles of $S_{n}$ are covered by the maximal intransitive subgroups of $S_{n}$ of type $(i, n-i)$ for $1 \leq i \leq[n / 3]$.

\section{Proof of Theorem 2}

In this whole section we will call $G:=A_{5} \succ C_{2}$, the semidirect product $\left(A_{5} \times\right.$ $\left.A_{5}\right) \rtimes\langle\varepsilon\rangle$ where $\varepsilon$, of order 2 , acts on $A_{5} \times A_{5}$ exchanging the two variables. Recall that the maximal subgroups of $G$ are of the following five types:

- The socle $N=A_{5} \times A_{5}$.

- Type 'r': $N_{G}\left(M \times M^{l}\right)$ where $l \in A_{5}$ and $M$ is a point stabilizer.

- Type 's': $N_{G}\left(M \times M^{l}\right)$ where $l \in A_{5}$ and $M$ is the normalizer of a Sylow 5 -subgroup.

- Type 't': $N_{G}\left(M \times M^{l}\right)$ where $l \in A_{5}$ and $M$ is an intransitive subgroup of type $(3,2)$.

- Type 'd': $N_{G}\left(\Delta_{\alpha}\right)$ where $\alpha \in S_{5}$ and $\Delta_{\alpha}:=\left\{\left(x, x^{\alpha}\right) \mid x \in A_{5}\right\}$.

Recall that:

- $N \cap N_{G}(H)=H$ for every $H$ of the type $M \times M^{l}$ or $\Delta_{\alpha}$ with $M$ a maximal subgroup of $A_{5}$.

- The element $(x, y) \varepsilon$ belongs to $N_{G}\left(M \times M^{l}\right)$ if and only if $x l^{-1}, l y \in M$. In particular $x y \in M$.

- The element $(x, y) \varepsilon$ belongs to $N_{G}\left(\Delta_{\alpha}\right)$ if and only if $(\alpha y)^{2}=x y$.

Let $\mathcal{M}$ be a family of proper subgroups of $G$ which cover $G$.

Observation 3. $N \in \mathcal{M}$

Proof. Let $x \in A_{5}$ be a 5-cycle, and let $y \in A_{5}$ be a 3-cycle. Then the element $(x, y)$ does not belong to any $N_{G}\left(M \times M^{l}\right)$ or $N_{G}\left(\Delta_{\alpha}\right)$ by the remarks above (no maximal subgroup of $A_{5}$ has order divisible by 3 and 5 ).

Call $i$ the number of subgroups of type $i$ in $\mathcal{M}$ for $i=r, s, t, d$.

The 'type' of an element $(x, y) \varepsilon \in G-N$ is the cyclic structure of the element $x y \in A_{5}$. The four possible cyclic structures will be denoted by $1,(3),(5),(2,2)$.

The only maximal subgroups of $G$ containing elements of type (3) are the ones of type $r$ or $t$ or $d$. A subgroup of type $r$ contains 96 elements of type (3). A subgroup of type $t$ contains 12 elements of type (3). A subgroup of type $d$ contains 20 elements of type (3). $G$ contains 1200 elements of type (3). In particular $96 r+12 t+20 d \geq 1200$, in other words

$$
24 r+3 t+5 d \geq 300 \text {. }
$$

The only maximal subgroups of $G$ containing elements of type (5) are the ones of type $s$ or $d$. A subgroup of type $s$ contains 40 elements of type (5). A subgroup of type $d$ contains 24 elements of type (5) if $\alpha$ is even, 0 if $\alpha$ is odd. $G$ contains 1440 elements of type (5). In particular $40 s+24 d \geq 1440$, in other words

$$
5 s+3 d \geq 180 .
$$


We know that $G$ admits a cover which consists of 57 proper subgroups, with $s=36, r=20, t=d=0$ (the 20 subgroups of type $r$ are $N_{G}\left(M \times M^{l}\right)$ where $l \in A_{5}$ and $\left.M \in\{\operatorname{Stab}(1), \operatorname{Stab}(2), \operatorname{Stab}(3), \operatorname{Stab}(4)\}\right)$.

Suppose by contradiction that $\sigma(G)<57$, and let $\mathcal{M}$ be a cover with 56 proper subgroups. In particular $r+s+t+d+1=56$, i.e. $r+s+t+d=55$.

Observation 4. $d \leq 33, s \geq 17$ and $r \geq 6$.

Proof. Inequality 2 re-writes as $s \geq 36-\frac{3}{5} d$. Since $r+s+t+d=55, r+t=$ $55-s-d \leq 55-36+\frac{3}{5} d-d=19-\frac{2}{5} d$. Combining this with inequality 1 we obtain $24\left(19-\frac{2}{5} d\right)+5 d \geq 300$, i.e. $d \leq 156 \cdot 5 / 23$, i.e. $d \leq 33$. Therefore $s \geq 36-\frac{3}{5} d=36-\frac{99}{5}>16$.

Inequality 1re-writes as $21 r+2 d-3 s+3(r+t+d+s) \geq 300$, i.e. $21 r+2 d-3 s \geq$ 135. Since $d \leq 33$ and $s \geq 17,21 r \geq 135+3 \cdot 17-2 \cdot 33=120$, i.e. $r \geq 6$.

Observation 5. $r+t+d \geq 20$ and $s<36$.

Proof. Consider the following elements of $A_{5}: a_{1}:=(243) \in \operatorname{Stab}(1), a_{2}:=(143) \in$ $\operatorname{Stab}(2), a_{3}:=(142) \in \operatorname{Stab}(3), a_{4}:=(132) \in \operatorname{Stab}(4)$. Let $\mathcal{X}$ be the set of elements of $G$ of the form $(x, y) \varepsilon$ with $x y=a_{i}$ for an $i \in\{1,2,3,4\}$ and $x \in J_{i}$, where $J_{i}$ is a fixed set of representatives of the right cosets of $\operatorname{Stab}(i)$, which will be specified later. Let $\mathcal{H}$ be the set of the 20 subgroups $N_{G}\left(M \times M^{l}\right)$ of $G$ of type $r$ with $M$ the stabilizer of $i$ for $i \in\{1,2,3,4\}$. Notice that every element of $\mathcal{X}$ lies in exactly one element of $\mathcal{H}$. Now observe that if a subgroup $N_{G}\left(K \times K^{l}\right)$ of type $t$ contains an element $(x, y) \varepsilon \in \mathcal{X}$ then $K$ is determined by $a_{i}=x y$ - use this to label the $K$ 's as $K_{i}$ for $i \in\{1,2,3,4\}-$, so that the only freedom is in the choice of the coset $K_{i} l$. We will choose the sets $J_{i}$ in such a way that any two elements of $J_{i}$ lie in different right cosets of $K_{i}$. This implies that for every subgroup $N_{G}\left(K \times K^{l}\right)$ of $G$ of type $t$ we have $\left|\mathcal{X} \cap N_{G}\left(K \times K^{l}\right)\right| \leq 1$. Let us choose the $J_{i}$ 's in such a way that for every subgroup $N_{G}\left(\Delta_{\alpha}\right)$ of type $d$ we have $\left|\mathcal{X} \cap N_{G}\left(\Delta_{\alpha}\right)\right| \leq 1$. Choose:

$$
\begin{aligned}
J_{1}= & \{(452),(12534),(13425),(14)(35),(23)(15)\}, \\
& J_{2}=\{(134),(245),(123),(152),(125)\}, \\
& J_{3}=\{(142),(132),(134),(153),(135)\}, \\
& J_{4}=\{(132),(142),(243),(154),(145)\} .
\end{aligned}
$$

We have that for any $i=1,2,3,4$ any two elements of $J_{i}$ lie in different right cosets of $K_{i}$. We have to check that every subgroup of the form $N_{G}\left(\Delta_{\alpha}\right)$ contains at most one element of $\mathcal{X}$. In other words we have to check that if $(x, y) \varepsilon \in \mathcal{X} \cap N_{G}\left(\Delta_{\alpha}\right)$ then $(x, y) \varepsilon$ is determined. We have $(\alpha y)^{2}=x y$, so that if $\alpha$ is even then $\alpha=x y x$, if $\alpha$ is odd then $\alpha=\tau_{x y} x y x$, where $\tau_{x y}$ is the transposition whose support is pointwise fixed by $x y$. Let

$$
P_{i}:=\left\{x y x \mid x y=a_{i},(x, y) \varepsilon \in \mathcal{X}\right\} \cup\left\{\tau_{x y} x y x \mid x y=a_{i},(x, y) \varepsilon \in \mathcal{X}\right\} \subset S_{5}
$$

for $i=1,2,3,4$. Clearly $\left|P_{i}\right|=10$ for $i=1,2,3$, 4 . All we have to show is that the $P_{i}$ 's are pairwise disjoint. This follows from the computation:

$$
\begin{gathered}
P_{1}=\{(25)(34),(12)(35),(135),(14532),(15)(24), \\
(125)(34),(1352),(35),(132)(45),(24)\}, \\
P_{2}=\{1,(15243),(14)(23),(14352),(14325), \\
(25),(1543),(14)(253),(1435),(1432)\},
\end{gathered}
$$




$$
\begin{gathered}
P_{3}=\{(124),(14)(23),(234),(14253),(14235), \\
(124)(35),(14)(235),(2354),(1425),(1423)\}, \\
P_{4}=\{(123),(13)(24),(12)(34),(13254),(13245), \\
(123)(45),(13)(245),(12)(345),(1325),(1324)\} .
\end{gathered}
$$

Clearly, the subgroups of $G$ of type $s$ do not contain any element of $\mathcal{X}$.

All this implies that $\mathcal{H}$ is definitely unbeatable on $\mathcal{X}$, hence $r+t+d \geq|\mathcal{H}|=20$. It follows that $56=|\mathcal{M}|=1+r+s+t+d>s+20$, i.e. $s<36$.

Observation 6. Let $M$ be the normalizer of a Sylow 5-subgroup of $A_{5}$, let $l \in A_{5}$ and suppose that $N_{G}\left(M \times M^{l}\right) \notin \mathcal{M}$. Then $N_{G}\left(\Delta_{\alpha}\right) \in \mathcal{M}$ for every $\alpha \in M l$. In particular if $\mathcal{L}$ is the family of the cosets $M l$ where $M<A_{5}$ is the normalizer of a Sylow 5-subgroup and $N_{G}\left(M \times M^{l}\right) \notin \mathcal{M}$ then the number of subgroups of type $d$ in $\mathcal{M}$ is at least the size of the union of $\mathcal{L}$.

Proof. The number of elements of type (5) in $N_{G}\left(M \times M^{l}\right)$ is 40 . Moreover the only maximal subgroup of $G$ of type $r, s, t$ which contains one of these 40 elements is the one we are considering: $x y \in M$ determines $M$ and $x \in M l$ determines $M l$. Let $c \in M$ be a 5 -cycle. The element $\left(x, x^{-1} c\right) \varepsilon$ belongs to $N_{G}\left(\Delta_{\alpha}\right)$ if and only if $\left(\alpha x^{-1} c\right)^{2}=c$, i.e. $\alpha x^{-1} c=c^{3}$, i.e. $\alpha=c^{2} x$. The result follows.

Lemma 9. We have the following facts:

(1) Let $k$ be a positive integer, and let $\mathcal{L}$ be the family of the cosets of the normalizers of the Sylow 5-subgroups of $A_{5}$. Then any subfamily of $\mathcal{L}$ consisting of exactly $k$ cosets covers at least $10 k-2\left(\begin{array}{c}k \\ 2\end{array}\right)$ elements of $A_{5}$.

(2) Let $H \neq K$ be two normalizers of Sylow 5-subgroups of $A_{5}$. Then for any $a_{1}, a_{2}, a_{3}, b_{1}, b_{2}, b_{3} \in A_{5}$ such that $H a_{1}, H a_{2}, H a_{3}, K b_{1}, K b_{2}, K b_{3}$ are pairwise distinct, the union

$$
H a_{1} \cup H a_{2} \cup H a_{3} \cup K b_{1} \cup K b_{2} \cup K b_{3}
$$

has size at least 42.

Proof. Let $H a, K b \in \mathcal{L}$. If the intersection $H a \cap K b$ is non-empty then it contains an element $x$, so that $H a=H x, K b=K x$, and $H a \cap K b=H x \cap K x=(H \cap K) x$. It follows that the maximum size of the intersection of two elements of $\mathcal{L}$ equals the maximum size of the intersection of two normalizers of Sylow 5 -subgroups, i.e. 2. Maximizing the sizes of the intersections we find that $k$ cosets cover at least $10 k-2\left(\begin{array}{c}k \\ 2\end{array}\right)$ elements.

We now prove the second statement. Clearly $\left|H a_{1} \cup H a_{2} \cup H a_{3}\right|=30$. Since $\left|H a_{i} \cap K b_{j}\right| \leq 2$ for every $i, j=1,2,3$,

$$
\left|H a_{1} \cup H a_{2} \cup H a_{3} \cup K b_{1} \cup K b_{2} \cup K b_{3}\right| \geq 30+3 \cdot(10-3 \cdot 2)=42,
$$

as we wanted.

Corollary 3. $s \leq 31$ and $d \geq 30$.

Proof. Recall that the subgroups of $G$ of type $s$ are 36. In the following we use Lemma 9 and Observation 6. If $s=32$ then $d \geq 28$, impossible; if $s=33$ then $d \geq 24$, impossible; if $s=34$ then $d \geq 18$, impossible since $r \geq 6$. Assume now $s=35$, so that $d \geq 10$. Since $r \geq 6,6+35+t+d \leq r+s+t+d=55$, i.e. $t+d \leq 14$. Thus inequality 1 implies that $5 \cdot 14 \geq 300-24 r$, i.e. $r \geq 10$. Hence $d=r=10$ and $t=0$. This contradicts inequality 1 Since $s<36$, we deduce that $s \leq 31$ and consequently $d \geq 30$. 
Since $d \geq 30, r+s+t+30 \leq r+s+t+d=55$, i.e. $r+s+t \leq 25$. Since $s \geq 17$ we obtain that $r+t \leq 8$. In particular $r \in\{6,7,8\}$.

- $r=6$. Then by inequality 1 we have $144+5(t+d) \geq 24 r+3 t+5 d=$ $24 r+3 t+5 d \geq 300$, and we deduce that $t+d \geq 32$. Therefore $55=$ $r+s+t+d \geq 6+s+32$, i.e. $s \leq 17$. Since $s \geq 17$ we obtain that $s=17$. Inequality 2 says that $5 \cdot 17+3 d \geq 180$, i.e. $d \geq 32$, so that $d=32$ and $t=0$.

- $r=7$. Since $d \geq 30,7+s+30 \leq r+s+t+d=55$, i.e. $s \leq 18$.

$-s=18$. Then $7+18+t+d=r+s+t+d=55$, i.e. $t+d=30$. Since $d \geq 30$ we obtain $d=30$ and $t=0$.

$-s=17$. Inequality 2 says that $5 \cdot 17+3 d \geq 180$, i.e. $d \geq 32$, so that $55=r+s+t+d \geq 7+17+32=56$, contradiction.

- $r=8$. Then since $r+s+t \leq 25$ we obtain $s+t \leq 17$, and since $s \geq 17$ we have $s=17, t=0$ and $d=30$. This contradicts inequality 2 ,

We deduce that either $(r, s, t, d)=(7,18,0,30)$ or $(r, s, t, d)=(6,17,0,32)$.

In both these cases there are at least 18 subgroups of type $s$ outside $\mathcal{M}$. Therefore Observation 6 and Lemma 9(2) imply that $d \geq 42$, a contradiction.

\section{REFERENCES}

[1] A. Ballester-Bolinches, L. M. Ezquerro, "Classes of Finite Groups", Springer, 2006.

[2] J.R. Britnell, A. Evseev, R.M. Guralnick, P.E. Holmes, A. Maróti, "Sets of elements that pairwise generate a linear group", Journal of Combinatorial Theory, 2006.

[3] R.A. Bryce, V. Fedri, L. Serena, "Subgroup coverings of some linear groups", Bull. Austral. Math. Soc., 60(1999), 227-238.

[4] P.J. Cameron, "Permutation Groups", London Mathematical Society Student Texts 45. Cambridge University Press, 1999.

[5] J.H.E. Cohn, "On n-sum groups", Math. Scand., 75(1) (1994), 44-58.

[6] J. H. Conway, R. T. Curtis, S. P. Norton, R. A. Parker, R. A. Wilson, "Atlas of finite groups", Clarendon Press Oxford.

[7] M. Garonzi, "Finite Groups that are Union of at most 25 Proper Subgroups", Journal of Algebra and its Applications, ISSN: 0219-4988.

[8] A. Lucchini, E. Detomi, "On the Structure of Primitive $n$-Sum Groups", CUBO A Mathematical Journal Vol.10 n. 03 (195-210), Ottobre 2008.

[9] A. Lucchini, A. Maróti, "On finite simple groups and Kneser graphs". J. Algebraic Combin. 30 (2009), no. 4, 549566.

[10] Lucido, M.S., "On the covers of finite groups". Groups St. Andrews 2001 in Oxford. Vol. II, 395399, London Math. Soc. Lecture Note Ser., 305, Cambridge Univ. Press, Cambridge, 2003, 20D60.

[11] A. Maroti, "On the orders of primitive groups". J. Algebra 258 (2002), no.2, 631-640.

[12] A. Maroti, "Covering The Symmetric Groups With Proper Subgroups", J. Combin. Theory Ser. A, 110(1) (2005), 97-111.

[13] A. Maroti, M. Garonzi, "Covering certain wreath products with proper subgroups", Journal of Group Theory, ISSN: 1433-5883.

[14] G. Scorza, "I gruppi che possono pensarsi come somme di tre loro sottogruppi", Boll. Un Mat. Ital. (1926) 216-218.

[15] M.J. Tomkinson, "Groups as the union of proper subgroups", Math. Scand., 81(2) (1997), 191-198. 